\title{
CLOSED SYSTEM OF COUPLING EFFECTS IN GENERALIZED THERMO-ELASTOPLASTICITY
}

\author{
Z. ŚLODERBACH \\ Opole University of Technology \\ Faculty of Applications of Chemistry and Mechanics \\ 45-036 Opole, ul. Luboszycka 7, POLAND \\ E-mail: z.sloderbach@po.opole.pl
}

\begin{abstract}
In this paper, the field equations of the generalized coupled thermoplasticity theory are derived using the postulates of classical thermodynamics of irreversible processses. Using the Legendre transformations two new thermodynamics potentials $P$ and $S$ depending upon internal thermodynamic forces $\Pi$ are introduced. The most general form for all the thermodynamics potentials are assumed instead of the usually used additive form. Due to this assumption, it is possible to describe all the effects of thermomechanical couples and also the elastic-plastic coupling effects observed in such materials as rocks, soils, concretes and in some metalic materials. In this paper not only the usual postulate of existence of a dissipation qupotential (the Gyarmati postulate) is used to derive the velocity equation. The plastic flow constitutive equations have the character of non-associated flow laws even when the Gyarmati postulate is assumed. In general formulation, the plastic strain rate tensor is normal to the surface of the generalized function of plastic flow defined in the the space of internal thermodynamic forces $\Pi$ but is not normal to the yield surface. However, in general formulation and after the use the Gyarmati postulate, the direction of the sum of the plastic strain rate tensor and the coupled elastic strain rate tensor is normal to the yield surface.
\end{abstract}

Key words: thermodynamic potentials, couplings effects, stresses, deformations, temperature, entropy, enthalpy, constitutive and rate equations, yield surface, plastic potential.

\section{Introduction}

The paper is a generalization of papers (Śloderbach [1], [2], [3]). This generalization consists in a formal derivation of two new thermodynamic potentials $P$ and $S$ dependent on the set internal thermodynamic forces $\Pi$ with the use of Legendre transformations. Owing to that, it was possible to close the system of physical thermo-elasto-plastic couplings (thermo-mechanical and elasto-plastic couplings). Suitable couplings were presented as a closed diagram of couplings. Moreover, derivation of a new thermodynamic potential $P$ allowed a precise description of values of the stored energy of plastic deformation (SEPD), (see Bever and Holt [4]; Oliferuk et al. [5], [6]; Śloderbach and Rechul [7]; Śloderbach and Pająk [8]) obtained during experiments (Oliferuk et al. [5], [6]) and specification of the equation of evolution for internal thermodynamic forces $\pi$, and determination of their initial values. The thermodynamic method of preparation space (Perzyna, [9]) was used for this purpose. The literature on coupled thermoelasto-plasticity is very vast and here we present only some selected problems, mainly those closely related to our present paper. The first papers concerning non-isothermal coupled thermo-elasto-plasticity can be found in Lehmann [10], Perzyna [9], Prager [11], Raniecki [12], [13], Raniecki and Sawczuk [14], [15], Raniecki and Thermann [16], Śloderbach [1], [2], Ziegler [17]. In all those papers, the displacement and velocity gradients were small, which means that the assumptions of the infinitesimal theory of the continuum were used. The first unclosed diagrams of couplings of thermo-elasto-plasticity can be found in Raniecki [12], [13], Raniecki and Sawczuk [14], Śloderbach [1]. The first closed diagrams of couplings and equations of thermo-elasticity were presented in Nye [18] and those for thermo-elasto-electro-magnetic effects in Newnham [19]. 
The selected effects of couplings of thermo-elasto-plasticity for large deformations (no closed system and diagram of couplings) can be found in Benall and Bigoni [20], Candija and Brnic [21], Casey [22], Lehmann [23], Nguyen [24], Perzyna [9], Raniecki and Thermann [16], Śloderbach and Pająk [25]. Most of the papers concerning large deformations described associated laws of plastic flow (see e.g., Benall and Bigoni [20], Candija and Brnic [21], Casey [22], Lehmann [23], Nguyen [24], Perzyna [9], Raniecki and Thermann [16]). Nguyen in paper [24], tried to specify some thermo-mechanical couplings in the range of large elasto-plastic deformations of metallic materials being under the influence of high pressures and quasistatic and dynamic loadings. Thus, derivation of the full and closed set of equations for couplings of thermo-elasto-plasticity for the case of large deformations and closure of the diagram of couplings (like in this paper) still remains an open scientific problem for future researchers.

In this paper, field equations of the generalized copuled theory of thermo-elasto-plasticity will be derived making use of the notions of classical thermodynamics of irreversible processes (Callen [26], Dillon [27], Gumiński [28], Kestin [29], Perzyna [9], Petryk [30]). The concept of macroscopic internal parameters will be used to describe the thermodynamic state of a thermo-elestic-plastic body (Dillon [27], Kestin [29], Perzyna [9], Raniecki [12]; [13], Śloderbach [1]; [2]). The above papers concerning similar problems will be used as a model. All the thermodynamic potentials are presented in general forms (not in additive forms) and as a result it is possible to describe the effects of elastic-plastic coupling observed in non-metal bodies such as, for instance, soils, rocks, concrete and other porous materials (Hueckel [31]).

Section 2 is devoted to the fundamental assumptions concerning the equation of state (the Gibbs equation) and the source of entropy. Discussion of general thermostatic properties of elastic-plastic bodies is presented in point 3 . This chapter also presents the basic thermodynamic identities including coupling effects.

The next parts contain the velocity equations of plastic flow. The evolution equations are also formulated for internal parameters on the basis of a general approach and a concept resulting from assumption of the Gyarmati postulate (Gyarmati [32]). The equations of evolution have been formulated for internal parameters on the basis of the general approach (not including potentials for the flows thermodynamic streams), (see Dillon [27], Lehmann [23], Perzyna [9], Pęcherski [33], Prager [11], Śloderbach [1], [2]) and the idea resulting from assumption of the Gyarmati postulate (Gyarmati [32], Petryk [30], Raniecki [12], [13], Raniecki and Thermann [16], Śloderbach [1], [2], [34]; Śloderbach and Rechul [7], Śloderbach and Pająk [25]).

During derivation of general rate equations, the frequently applied postulate about the occurrence of the thermodynamic potential (Gyarmati postulate) is not used. As in papers (Śloderbach [1], [2]), the constitutive equations of plastic flow are non-associated even in a specific case when the Gyarmati postulate is assumed (Gyarmati [32]). Application of that postulate need not lead to associated rules of plastic flow. However, its application causes that a direction of the sum of tensors of plastic strain rates and rates of irreversible plastic strains and rates of irreversible strains caused by plastic strains (effect of the elasticplastic coupling) is perpendicular to the instantaneous area of plasticity. The occurrence of the elastic-plastic coupling effect allows the application of those equations to describe the behaviour of metallic materials under isothermic and non-isothermic conditions, and porous materials, sinters, rocks and soils (Hueckel [31], Śloderbach [1], [2]).

Neither the usual postulate of existence of dissipation potential (the Gyarmati postulate) is used to derive the velocity equations. Similarly as in the papers (Śloderbach [1], [2], [3], Śloderbach and Pająk [25]) the equations of plastic flow have non-associated forms. The use of that postulate does not necessarily lead to the associated laws of plastic flow. If turns out, however, that, as a consequence of that assumption, the direction of the sum of the plastic strain rate tensor and the coupled part of elastic strain tensor is normal to the instantaneous surface of plasticity.

It will be assumed throughout the entire paper that the displacement and velocity gradients are small, which means that the assumptions of the infinitesimal theory of the continuum are used. It is assumed for simplicity that all the equations and the entire description are expressed in rectangular Cartesian coordinates. 


\section{Fundamental assumptions. The Gibbs equation and the source of entropy}

Let us assume that the local thermodynamic state is described by the following parameters of state (Raniecki [13], Raniecki and Sawczuk [14], Śloderbach [1], [2], [3])

$\varepsilon^{\mathrm{e}}-$ tensor of small elastic strain,

$s$ - specific entropy (per unit mass),

$\boldsymbol{\kappa}^{(N)}-$ set of symmetric internal tensor parameters of second order $(N=1, \ldots, n)$, $\boldsymbol{\kappa}=\boldsymbol{\kappa}^{\mathrm{T}}$, that is $\kappa_{i j}=\kappa_{j i}$,

$\kappa^{(M)}-$ set of internal scalar parameters $(M=1, \ldots, m)$.

Now the symbol $K$ will denote the set of internal parameters in the form of a vector of pair $K \Leftrightarrow$ $\left\{\boldsymbol{\kappa}^{(M)}, \kappa^{(M)}\right\},(N=1, \ldots n)$ and $(M=1, \ldots, m)$.

Let us assume that the fundamental equation of state, i.e., the Gibbs equation, has the following form of a differential of the specific internal energy $U$ (Śloderbach [1], [2])

$$
d U\left(s, \varepsilon^{e}, K\right)=T d s+\frac{1}{\rho_{0}} \sigma: d \varepsilon^{e}+\frac{1}{\rho_{0}} \Pi \cdot d K
$$

where $\Pi$ is now the vector pair of the internal set thermodynamic forces associated with the set of internal parameters $K$, where $\Pi \Leftrightarrow\left\{\pi^{(\mathrm{N})}, \pi^{(M)}\right\},(N=1, \ldots n)$ and $(M=1, \ldots, m)$.

The local approach to the principle of conservation of energy is as follows (Raniecki [13], Śloderbach [1], [2])

$$
\dot{U}=\frac{1}{\rho_{0}} \sigma: \dot{\varepsilon}-\frac{1}{\rho_{0}} \operatorname{div} \mathbf{q}
$$

where $\operatorname{div} \mathbf{q}=\frac{\partial q_{i}}{\partial x i}, x_{i}$ - orthogonal coordinates which express the initial location of the particle. The equation of local entropy balance per unit volume of the body has the form (Raniecki [13], Śloderbach [1], [2])

$$
\rho_{0} \dot{s}=-\operatorname{div}\left(\frac{\mathbf{q}}{T}\right)+\delta^{s}
$$

where $\left(\frac{\mathbf{q}}{T}\right)$ - is the entropy flux and $\delta^{s}$ is the entropy produced in a definite particle per unit time and volume.

The local formulation of the second law of thermodynamics is given by the inequality

$$
\delta^{s} \geq 0
$$

The entropy production can be evaluated by solving a set of three Eqs (2.1) to (2.3) for $\left(\dot{U}, \dot{s}\right.$ and $\left.\delta^{s}\right)$

$$
T \delta^{s}=D-\frac{1}{T} \mathbf{q}: \nabla T, \quad \nabla T=\operatorname{grad} T
$$

where $D$ - expresses dissipation of mechanical energy per unit time and volume 
and

$$
D=\sigma: \dot{\varepsilon}^{p}-\Pi \cdot \dot{K} \geq 0,
$$

$$
\dot{\boldsymbol{\varepsilon}}=\dot{\boldsymbol{\varepsilon}}^{e}+\dot{\boldsymbol{\varepsilon}}^{p} .
$$

The set of forces $n$ (2.5) and (2.6)

$$
X^{d}=\left\{\sigma,-\Pi, \frac{1}{T} \operatorname{grad} T\right\},
$$

is referred to as a set of dissipation forces or a set of thermodynamic impulses, and

$$
\dot{x}^{d}=\left\{\dot{\boldsymbol{\varepsilon}}^{p}, \dot{K}, \mathbf{q}\right\},
$$

is a set of measures of thermodynamic flow rates joined with the set Eq.(2.8).

\section{Discussion of the thermostatic properties of an elastic-plastic body}

Some different thermodynamic potentials may be used for the description of the thermo thermostatic properties of material. This leads, of course, to equivalent descriptions.

Apart from three classical potentials, i.e., internal energy, free energy (Helmholz function) and Gibbs potential (Gibbs function), two additional potentials dependent on the internal thermodynamic forces $\Pi$ have been derived using the Legendre transformation.

In this paper, the authors use the following parameters of state: $\left[(s, T),\left(\varepsilon^{\mathrm{e}}, \sigma\right),(K, \Pi)\right]$. We can distinguish eight subsets from that set, containing suitable three parameters of state: $\left\{s, \varepsilon^{\mathrm{e}}, K\right\},\left\{s, \varepsilon^{\mathrm{e}}, \Pi\right\},\{s$, $\sigma, K\},\{s, \sigma, \Pi\},\left\{T, \varepsilon^{\mathrm{e}}, K\right\},\left\{T, \varepsilon^{\mathrm{e}}, \Pi\right\},\{T, \sigma, K\},\{T, \sigma, \Pi\}$. The first five subsets will be used as parameters of state for the corresponding five thermodynamic potentials. That system of five independent parameters of state makes it possible to assume the diagram of thermo-elasto-plastic couplings as a closed diagram having features of transitivity.

Let us denote the sets of five independent parameters of state as follows

$$
\begin{array}{ll}
Y_{K}^{s \varepsilon}=\left\{s, \varepsilon^{e}, K\right\}, & Y_{K}^{T \varepsilon}=\left\{T, \varepsilon^{e}, K\right\}, \quad Y_{K}^{T \sigma}=\{T, \sigma, K\}, \\
Y_{\Pi}^{T \varepsilon}=\left\{T, \varepsilon^{e}, \Pi\right\}, \quad Y_{\Pi}^{T \sigma}=\{T, \sigma, \Pi\} .
\end{array}
$$

The first three sets are widely used (Raniecki [13], Raniecki and Sawczuk [14], Raniecki and Thermann [16], Śloderbach [1], [2]) and they are independent variable quantities in three potentials: internal energy, free energy and the Gibbs function, respectively. The fourth set was introduced in Śloderbach and Rechul [7] in order to specify a new potential $P$ useful in description of the stored energy of plastic deformation (SEPD) (see Bever and Holt [4], Oliferuk et al. [5], Oliferuk [6], Śloderbach and Rechul [7], Śloderbach and Pająk [8]). The fifth set was introduced in this paper for the first time in order to specify a closed system of thermomechanical couplings and the elastic-plastic coupling in thermo-elasto-plasticity, as well as in order to close of the graphical diagram of couplings (Raniecki [12], [13], Śloderbach [1], [2]).

The relevant thermodynamic potentials suitable for sets of independent parameters of state (3.1) will be denoted as follows

$$
U=U\left(Y_{K}^{S E}\right) \text {-internal energy, }
$$




$$
\begin{aligned}
& A=A\left(Y_{K}^{T \varepsilon}\right)=[U-T s]_{s=s\left(Y_{K}^{s \varepsilon}\right)} \text { - free energy (Helmholtz function), } \\
& \left.G=G\left(Y_{K}^{T \sigma}\right)=\left[A-\frac{1}{\rho_{0}} \sigma \cdot \varepsilon^{e}\right]_{\varepsilon=\varepsilon}\right]^{e}\left(Y_{K}^{T \sigma}\right) \text { - Gibbs function, } \\
& P=P\left(Y_{\Pi}^{T \varepsilon}\right)=\left[A-\frac{1}{\rho_{0}} \Pi \cdot K\right]_{K=K\left(Y_{\Pi}^{T \varepsilon}\right)} \text { - potential depending on set } Y_{\Pi}^{T \varepsilon},
\end{aligned}
$$

(see Śloderbach and Rechul [7])

$$
S=S\left(Y_{\Pi}^{T \sigma}\right)=\left[G-\frac{1}{\rho_{0}} \Pi \cdot K\right]_{K=K\left(Y_{\Pi}^{T \sigma}\right)} \text { - new potential depending on set } Y_{\Pi}^{T \sigma}
$$

Their total differentials are

$$
\left\{\begin{array}{l}
d U=T d s+\frac{1}{\rho_{0}} \sigma: d \varepsilon^{e}+\frac{1}{\rho_{0}} \Pi \cdot d K \\
d A=-s d T+\frac{1}{\rho_{0}} \sigma: d \varepsilon^{e}+\frac{1}{\rho_{0}} \Pi \cdot d K \\
d G=-s d T-\frac{1}{\rho_{0}} \varepsilon^{e}: d \sigma+\frac{1}{\rho_{0}} \Pi \cdot d K \\
d P=-s d T+\frac{1}{\rho_{0}} \sigma: d \varepsilon^{e}-\frac{1}{\rho_{0}} K \cdot d \Pi \\
d S=-s d T-\frac{1}{\rho_{0}} \varepsilon^{e}: d \sigma-\frac{1}{\rho_{0}} K \cdot d \Pi
\end{array}\right.
$$

Thermal equations of state are given in Tab.1.

\begin{tabular}{|c|c|c|c|c|}
\hline $\begin{array}{c}U=U\left(Y_{K}^{s \varepsilon}\right) \\
(3.4)\end{array}$ & $\begin{array}{c}A=A\left(Y_{K}^{T \varepsilon}\right) \\
(3.5)\end{array}$ & $\begin{array}{c}G=G\left(Y_{K}^{T \sigma}\right) \\
(3.6)\end{array}$ & $\begin{array}{c}P=P\left(Y_{\Pi}^{T \varepsilon}\right) \\
(3.7)\end{array}$ & $\begin{array}{c}S=S\left(Y_{\Pi}^{T \sigma}\right) \\
(3.8)\end{array}$ \\
\hline 1 & 2 & 3 & 5 \\
\hline$T=\frac{\partial U\left(Y_{K}^{s \varepsilon}\right)}{\partial s}$ & $-S=\frac{\partial A\left(Y_{K}^{T \varepsilon}\right)}{\partial T}$ & $-S=\frac{\partial G\left(Y_{K}^{T \sigma}\right)}{\partial T}$ & $-S=\frac{\partial P\left(Y_{\Pi}^{T \varepsilon}\right)}{\partial T}$ & $-S=\frac{\partial S\left(Y_{\Pi}^{T \sigma}\right)}{\partial T}$ \\
\hline$\frac{1}{\rho_{0}} \sigma=\frac{\partial U\left(Y_{K}^{s \varepsilon}\right)}{\partial \varepsilon^{\mathrm{e}}}$ & $\frac{1}{\rho_{0}} \sigma=\frac{\partial A\left(Y_{K}^{T \varepsilon}\right)}{\partial \varepsilon^{e}}$ & $-\frac{\varepsilon^{e}}{\rho_{0}}=\frac{\partial G\left(Y_{K}^{T \sigma}\right)}{\partial \sigma}$ & $\frac{1}{\rho_{0}} \sigma=\frac{\partial P\left(Y_{\Pi}^{T \varepsilon}\right)}{\partial \varepsilon^{e}}$ & $\frac{1}{\rho_{0}} \varepsilon^{e}=\frac{\partial S\left(Y_{\Pi}^{T \sigma}\right)}{\partial \sigma}$ \\
\hline$\frac{1}{\rho_{0}} \Pi=\frac{\partial U\left(Y_{K}^{s \varepsilon}\right)}{\partial K}$ & $\frac{1}{\rho_{0}} \Pi=\frac{\partial A\left(Y_{K}^{T \varepsilon}\right)}{\partial K}$ & $\frac{1}{\rho_{0}} \Pi=\frac{\partial G\left(Y_{K}^{T \sigma}\right)}{\partial K}$ & $-\frac{1}{\rho_{0}} \mathrm{~K}=\frac{\partial P\left(Y_{\Pi}^{T \varepsilon}\right)}{\partial \Pi}$ & $-\frac{1}{\rho_{0}} K=\frac{\partial S\left(Y_{\Pi}^{T \sigma}\right)}{\partial \Pi}$ \\
\hline
\end{tabular}

The fundamental physical quantities describing the thermostatic properties of solids are defined as follows (Śloderbach [1], [2]) 


$$
\begin{aligned}
& c_{\varepsilon}\left(Y_{K}^{T \varepsilon}\right)=T \frac{\partial s\left(Y_{K}^{T \varepsilon}\right)}{\partial T}=\frac{\partial U\left(Y_{K}^{T \varepsilon}\right)}{\partial T}, \quad c_{\sigma}=T \frac{\partial s\left(Y_{K}^{T \sigma}\right)}{\partial T}, \\
& \boldsymbol{L}\left(Y_{K}^{T \sigma}\right)=\frac{\partial \boldsymbol{\varepsilon}^{e}\left(Y_{K}^{T \sigma}\right)}{\partial \sigma}, \quad \boldsymbol{M}\left(Y_{K}^{T \varepsilon}\right)=\frac{\partial \sigma\left(Y_{K}^{T \varepsilon}\right)}{\partial \boldsymbol{\varepsilon}^{e}}, \\
& \boldsymbol{Z}\left(Y_{K}^{T \sigma}\right)=\frac{\partial \boldsymbol{\varepsilon}^{e}\left(Y_{K}^{T \sigma}\right)}{\partial K}, \quad \boldsymbol{R}\left(Y_{\Pi}^{T \sigma}\right)=\frac{\partial \boldsymbol{\varepsilon}^{e}\left(Y_{\Pi}^{T \sigma}\right)}{\partial \Pi}, \\
& \boldsymbol{N}\left(Y_{K}^{T \varepsilon}\right)=\frac{\partial \sigma\left(Y_{K}^{T \varepsilon}\right)}{\partial K}, \quad \boldsymbol{S}\left(Y_{\Pi}^{T \varepsilon}\right)=\frac{\partial \boldsymbol{\sigma}\left(Y_{\Pi}^{T \varepsilon}\right)}{\partial \Pi}
\end{aligned}
$$

where

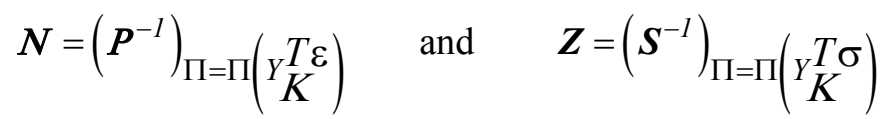

where $\boldsymbol{Z}$ - is the vector of pairs of tensors of the fourth and second order representing the isothermal variation of elastic deformation due to the internal processes accompanying plastic deformation in state $Y_{K}^{T \sigma}$. Physically, it means a change of Young's modulus caused by plastic deformations. Figures 2 and 3 show the linear and non-linear changes of Young's modulus and of elastic deformations with the stresses during the unloading processes. $\boldsymbol{N}$ - is the vector of pairs of tensors of the fourth and second order representing isothermal variation of the state of stress due to internal processes accompanying plastic deformation in the state $Y_{K}^{T \varepsilon}$.

The quantities (3.9) to (3.12) are not independent. They satisfy the set of the following identities resulting from the existence of thermodynamic potentials (Raniecki [12], [13], Raniecki and Sawczuk [14], Raniecki and Thermann [16], Śloderbach [1], [2])

$$
\begin{aligned}
& \boldsymbol{M}=\left(\boldsymbol{L}^{-1}\right)_{\sigma=\sigma}\left(Y_{K}^{T \varepsilon}\right), \\
& 2\left(M_{i j m n} L_{m n r s}\right)_{\boldsymbol{\sigma}=\sigma}\left(Y_{K}^{T \varepsilon}\right)=\delta_{i s} \delta_{j r}+\delta_{i r} \delta_{j s} \quad \text { and } \quad M_{i j m n}=M_{m n i j}=M_{j i m n}=M_{i j n m} . \\
& c_{\sigma}=\left(c_{\varepsilon}+\frac{T}{\rho_{0}} \boldsymbol{\alpha}: \boldsymbol{M} \boldsymbol{\alpha}\right)_{\boldsymbol{\varepsilon}=\varepsilon}\left(Y_{K}^{T \sigma}\right) \text { and } \boldsymbol{\alpha}\left(Y_{K}^{T \sigma}\right)=\frac{\partial \boldsymbol{\varepsilon}^{e}}{\partial T}, \quad \frac{1}{\rho_{0}} \frac{\partial \Pi\left(Y_{K}^{s \varepsilon}\right)}{\partial s}=\frac{\partial T\left(Y_{\mathrm{K}}^{s \varepsilon}\right)}{\partial K}, \\
& \frac{\partial s\left(Y_{K}^{T \varepsilon}\right)}{\partial \boldsymbol{\varepsilon}^{e}}=-\frac{1}{\rho_{0}} \frac{\partial \boldsymbol{\sigma}\left(Y_{K}^{T \varepsilon}\right)}{\partial T}=\frac{1}{\rho_{0}}(\boldsymbol{M} \boldsymbol{\alpha})_{\boldsymbol{\sigma}=\sigma}\left(Y_{K}^{T \varepsilon}\right), \quad-\rho_{0} \frac{\partial s\left(Y_{K}^{T \varepsilon}\right)}{\partial K}=\frac{\partial \Pi\left(Y_{K}^{T \varepsilon}\right)}{\partial T}, \\
& \frac{\partial s\left(Y_{K}^{T \sigma}\right)}{\partial \boldsymbol{\sigma}}=\frac{1}{\rho_{0}} \boldsymbol{\alpha}, \alpha_{i j}=\alpha_{j i}, \frac{\partial \boldsymbol{\varepsilon}^{e}\left(Y_{K}^{T \sigma}\right)}{\partial T}=\rho_{0} \frac{\partial s\left(Y_{K}^{T \sigma}\right)}{\partial \boldsymbol{\sigma}} \text { and }-\rho_{0} \frac{\partial s\left(Y_{K}^{T \sigma}\right)}{\partial K}=\frac{\partial \Pi\left(Y_{K}^{T \sigma}\right)}{\partial T} .
\end{aligned}
$$


The thermodynamic potentials being not expressed in an additive form but in the most general form (Śloderbach [1], [2], Śloderbach and Pająk [25]) give the following additional identities of thermostatic couplings, which will be used in a further part of the paper

$$
\begin{aligned}
& \left\{\begin{array}{l}
-\boldsymbol{L} \boldsymbol{N}=-\boldsymbol{L}\left(Y_{K}^{T \sigma}\right) \boldsymbol{N}\left(Y_{K}^{T \varepsilon}\right)=\boldsymbol{Z} \\
-\boldsymbol{M} \boldsymbol{Z}=-\boldsymbol{M}\left(Y_{K}^{T \varepsilon}\right) \boldsymbol{Z}\left(Y_{K}^{T \sigma}\right)=\boldsymbol{N}
\end{array}\right. \\
& \boldsymbol{N}=\boldsymbol{N}\left(Y_{K}^{T \varepsilon}\right)=\frac{\partial \boldsymbol{\sigma}\left(Y_{K}^{T \varepsilon}\right)}{\partial K}=\frac{\partial \Pi\left(Y_{K}^{T \varepsilon}\right)}{\partial \boldsymbol{\varepsilon}^{e}} \text { and } \boldsymbol{Z}=\boldsymbol{Z}\left(Y_{K}^{T \sigma}\right)=\frac{\partial \boldsymbol{\varepsilon}^{e}\left(Y_{K}^{T \sigma}\right)}{\partial K}=-\frac{\partial \Pi\left(Y_{K}^{T \sigma}\right)}{\partial \boldsymbol{\sigma}}
\end{aligned}
$$

and

$$
\begin{aligned}
& \frac{\partial s\left(Y_{K}^{T \sigma}\right)}{\partial K}=\frac{\partial s\left(Y_{K}^{T \varepsilon}\right)}{\partial K}+\frac{1}{\rho_{0}} \boldsymbol{\alpha} \circ(\boldsymbol{M Z}), \\
& \frac{\partial \Pi\left(Y_{K}^{T \sigma}\right)}{\partial T}=\frac{\partial \Pi\left(Y_{K}^{T \varepsilon}\right)}{\partial T}-\boldsymbol{\alpha} \circ(\boldsymbol{M Z}) .
\end{aligned}
$$

The identities (3.18), (3.20) and (3.21) are sometimes called thermodynamic identities of the second order. The identities expressing thermostatic couplings (3.19) have the following physical interpretation. A variation in internal forces $\Pi$ as a result of elastic strain results in a process of hardening (softening) of the materials, and variation in internal forces as a result of stress is connected with a variation in the moduli of elasticity as a result of variation in internal parameters, see Eqs (3.11) 1 and (3.11) $)_{3}$. The identities (3.18), (3.20) and (3.21) are complex identities and are sometimes referred to as identities of the second order.

The most important thermostatic properties of elastic-plastic materials can be discussed by assuming consecutively $\left(Y_{\mathrm{K}}^{T \varepsilon}, Y_{K}^{T \sigma}, Y_{\Pi}^{T \varepsilon}, Y_{\Pi}^{T \sigma}, Y_{K}^{s \sigma}, Y_{\mathrm{K}}^{s \varepsilon}, Y_{\Pi}^{s \varepsilon}, Y_{\Pi}^{s \sigma}\right)$ as a set of independent parameters of state and evaluating the increments in the dependent parameters [cf. columns 2, 3, 4 and 5 of Tab.1 and the formulae (3.9)-(3.21)]. Thus, we obtain the following expressions

$$
\left\{\begin{array}{l}
T d s\left(Y_{K}^{T \varepsilon}\right)=\gamma_{1} c_{\varepsilon} d T+\bar{\gamma}_{6} \frac{T}{\rho_{0}} \boldsymbol{\alpha M}: d \varepsilon^{e}-\bar{\gamma}_{8} \frac{T}{\rho_{0}} \frac{\partial \Pi}{\partial T} \cdot d K \\
T d s\left(Y_{K}^{T \sigma}\right)=\gamma_{1} c_{\sigma} d T+\gamma_{7} \frac{T}{\rho_{0}} \alpha: d \sigma-\bar{\gamma}_{8} \frac{T}{\rho_{0}} \frac{\partial \Pi}{\partial T} \cdot d K \\
T d s\left(Y_{\Pi}^{T \varepsilon}\right)=\gamma_{1} c_{\varepsilon} d T+\bar{\gamma}_{6} \frac{T}{\rho_{0}} \alpha \boldsymbol{M}: d \varepsilon^{e}+\bar{\gamma}_{14} \frac{\partial s\left(Y_{\Pi}^{T \varepsilon}\right)}{\partial \Pi} \cdot d \Pi \\
T d s\left(Y_{\Pi}^{T \sigma}\right)=\gamma_{1} c_{\sigma} d T+\gamma_{7} \frac{T}{\rho_{0}} \alpha: d \sigma+\bar{\gamma}_{14} \frac{\partial s\left(Y_{\Pi}^{T \sigma}\right)}{\partial \Pi} \cdot d \Pi .
\end{array}\right.
$$




$$
\begin{aligned}
& \int d T\left(Y_{K}^{s \varepsilon}\right)=\bar{\gamma}_{1} \frac{\partial T}{\partial s} d s+\bar{\gamma}_{4} \frac{\partial T}{\partial \varepsilon^{e}}: d \varepsilon^{e}+\bar{\gamma}_{15} \frac{\partial T}{\partial K} \cdot d K, \\
& d T\left(Y_{K}^{s \sigma}\right)=\bar{\gamma}_{1} \frac{\partial T}{\partial s} d s+\bar{\gamma}_{5} \frac{\partial T}{\partial \sigma}: d \sigma+\bar{\gamma}_{15} \frac{\partial T}{\partial K} \cdot d K, \\
& d T\left(Y_{\Pi}^{s \varepsilon}\right)=\bar{\gamma}_{1} \frac{\partial T}{\partial s} d s+\bar{\gamma}_{4} \frac{\partial T}{\partial \varepsilon^{e}}: d \varepsilon^{e}+\bar{\gamma}_{13} \frac{\partial T}{\partial \Pi} \cdot d \Pi, \\
& d T\left(Y_{\Pi}^{s \sigma}\right)=\bar{\gamma}_{1} \frac{\partial T}{\partial s} d s+\bar{\gamma}_{4} \frac{\partial T}{\partial \sigma}: d \sigma+\bar{\gamma}_{15} \frac{\partial T}{\partial \Pi} \cdot d \Pi, \\
& \left\{\begin{array}{l}
d \boldsymbol{\varepsilon}^{e}\left(Y_{K}^{T \sigma}\right)=\gamma_{4} \boldsymbol{\alpha} d T+\gamma_{2} \boldsymbol{L} d \boldsymbol{\sigma}+\bar{\gamma}_{9} \boldsymbol{Z} d K \\
d \boldsymbol{\varepsilon}^{e}\left(Y_{\Pi}^{T \sigma}\right)=\gamma_{4} \boldsymbol{\alpha} d T+\gamma_{2} \boldsymbol{L} d \boldsymbol{\sigma}+\bar{\gamma}_{11} \boldsymbol{R} d \Pi, \\
d \boldsymbol{\varepsilon}^{e}\left(Y_{K}^{s \sigma}\right)=\gamma_{6} \frac{\partial \boldsymbol{\varepsilon}^{e}}{\partial s} d s+\gamma_{2} \boldsymbol{L} d \boldsymbol{\sigma}+\bar{\gamma}_{9} \boldsymbol{Z} d K \\
d \boldsymbol{\varepsilon}^{e}\left(Y_{\Pi}^{s \sigma}\right)=\gamma_{6} \frac{\partial \boldsymbol{\varepsilon}^{e}}{\partial s} d s+\gamma_{2} \boldsymbol{L} d \boldsymbol{\sigma}+\bar{\gamma}_{11} \boldsymbol{R} d \Pi .
\end{array}\right. \\
& \int d \sigma\left(Y_{\mathrm{K}}^{T \varepsilon}\right)=\gamma_{5} \boldsymbol{M} \boldsymbol{\alpha} d T+\bar{\gamma}_{2} \boldsymbol{M} d \boldsymbol{\varepsilon}^{e}+\bar{\gamma}_{10} \boldsymbol{Z} d K, \\
& d \sigma\left(Y_{\Pi}^{T \varepsilon}\right)=\gamma_{5} \boldsymbol{M} \boldsymbol{\alpha} d T+\bar{\gamma}_{2} \boldsymbol{M} d \boldsymbol{\varepsilon}^{e}+\bar{\gamma}_{12} \boldsymbol{S} d \Pi, \\
& d \boldsymbol{\sigma}\left(Y_{\mathrm{K}}^{s \varepsilon}\right)=\bar{\gamma}_{7} \frac{\partial \boldsymbol{\sigma}}{\partial s} d s+\bar{\gamma}_{2} \boldsymbol{M} d \boldsymbol{\varepsilon}^{e}+\bar{\gamma}_{10} \boldsymbol{Z} d K, \\
& d \sigma\left(Y_{\Pi}^{T \varepsilon}\right)=\bar{\gamma}_{7} \frac{\partial \boldsymbol{\sigma}}{\partial s} d s+\bar{\gamma}_{2} \boldsymbol{M} d \boldsymbol{\varepsilon}^{e}+\bar{\gamma}_{12} \boldsymbol{S} d \Pi .
\end{aligned}
$$




$$
\left\{\begin{array}{l}
d \Pi\left(Y_{K}^{T \varepsilon}\right)=\gamma_{13} \frac{\partial \Pi}{\partial T} d T+\gamma_{11} \frac{\partial \Pi}{\partial \varepsilon^{e}} \circ d \varepsilon^{e}+\gamma_{3} \frac{\partial \Pi}{\partial K} \circ d K, \\
d \Pi\left(Y_{K}^{T \sigma}\right)=\gamma_{13} \frac{\partial \Pi}{\partial T} d T+\gamma_{12} \frac{\partial \Pi}{\partial \sigma} \circ d \sigma+\gamma_{3} \frac{\partial \Pi}{\partial K} \circ d K \\
d \Pi\left(Y_{K}^{s \varepsilon}\right)=\gamma_{14} \frac{\partial \Pi}{\partial s} d s+\gamma_{11} \frac{\partial \Pi}{\partial \varepsilon^{e}} \circ d \varepsilon^{e}+\gamma_{3} \frac{\partial \Pi}{\partial K} \circ d K \\
d \Pi\left(Y_{K}^{s \sigma}\right)=\gamma_{14} \frac{\partial \Pi}{\partial s} d s+\gamma_{12} \frac{\partial \Pi}{\partial \sigma} \circ d \sigma+\gamma_{3} \frac{\partial \Pi}{\partial K} \circ d K \\
d K\left(Y_{\Pi}^{T \varepsilon}\right)=\gamma_{15} \frac{\partial \mathrm{K}}{\partial T} d T+\gamma_{9} \frac{\partial \mathrm{K}}{\partial \varepsilon^{e}} \circ d \varepsilon^{e}+\bar{\gamma}_{3} \frac{\partial \mathrm{K}}{\partial \Pi} \circ d \Pi, \\
d K\left(Y_{\Pi}^{T \sigma}\right)=\gamma_{15} \frac{\partial \mathrm{K}}{\partial T} d T+\gamma_{10} \frac{\partial \mathrm{K}}{\partial \sigma} \circ d \sigma+\bar{\gamma}_{3} \frac{\partial \mathrm{K}}{\partial \Pi} \circ d \Pi, \\
d K\left(Y_{\Pi}^{s \sigma}\right)=\gamma_{8} \frac{\partial \mathrm{K}}{\partial s} d s+\gamma_{10} \frac{\partial \mathrm{K}}{\partial \sigma} \circ d \sigma+\bar{\gamma}_{3} \frac{\partial \mathrm{K}}{\partial \Pi} \circ d \Pi . \\
d K\left(Y_{\Pi}^{s \varepsilon}\right)=\gamma_{8} \frac{\partial \mathrm{K}}{\partial s} d s+\gamma_{9} \frac{\partial \mathrm{K}}{\partial \varepsilon^{e}} \circ d \varepsilon^{e}+\bar{\gamma}_{3} \frac{\partial \mathrm{K}}{\partial \Pi} \circ d \Pi,
\end{array}\right.
$$

Thermostatic couplings in the area of thermo-elasto-plastic interactions are presented in Fig.1. It uses similar but incomplete schemes of couplings presented in papers (Raniecki [12], [13], Raniecki and Sawczuk, 1973; Śloderbach [1]) or thermo-elastic couplings completed with the electric and magnetic fields, including, for example, phenomena of thermo-electric polarization (Newnham [19]).

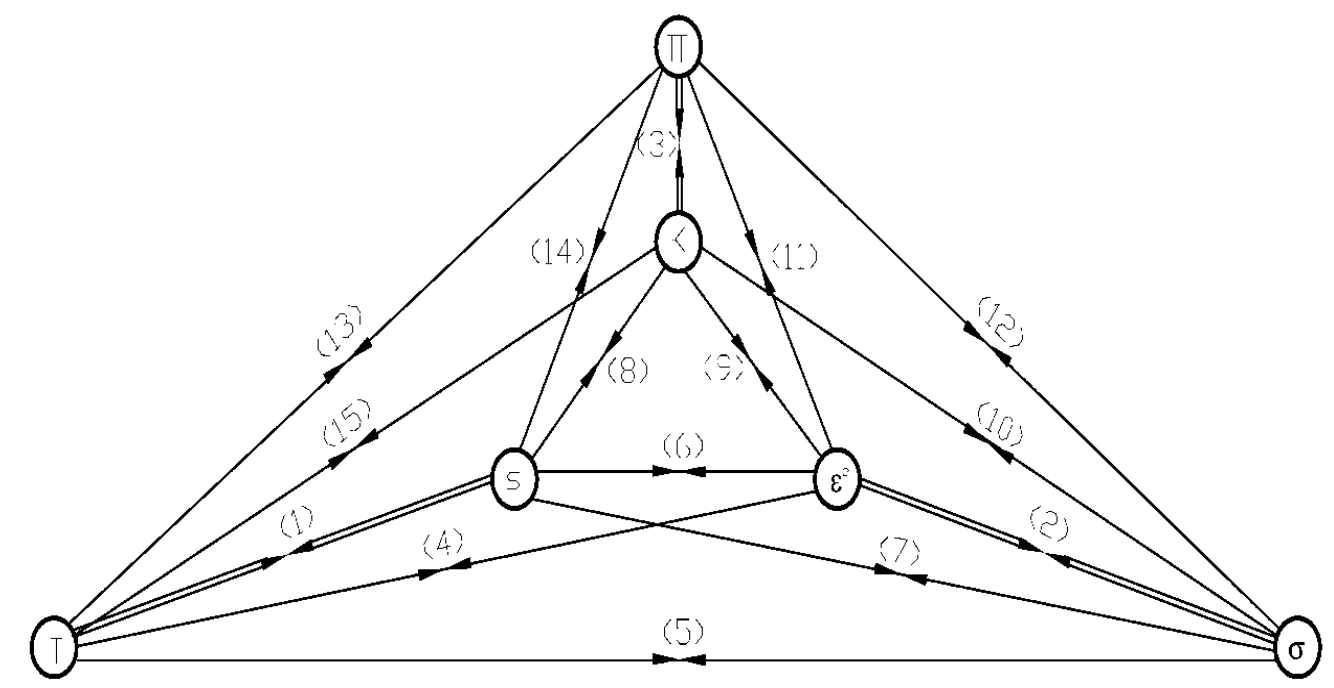

Fig.1. Closed diagram of couplings occurring in thermomechanics of elastic-plastic bodies. 
The diagram shows 15 possible coupled connections between two independent parameters of state. Particular types of thermodynamic, thermomechanical and mechanical (elastic-plastic) couplings are denoted as the Greek letter $\gamma$ with a suitable subscript or with subscript and dash. It was assumed that couplings with the upward (vertical or at an angle) and horizontal (to the right) arrows were expressed by the symbol $\gamma_{i}(i=$ $1,2, \ldots, 15)$ (with no dash). The couplings with downward arrows (vertical or at an angle) and horizontal (to the left) arrows were denoted by the symbol with subscript and dash $\bar{\gamma}_{i} \quad(i=1,2, \ldots, 15)$. Dimensionless coefficients $\gamma_{i}$ and $\bar{\gamma}_{i}$ have no physical meaning. The ideas presented in Raniecki and Sawczuk [14], Raniecki [12], [13], Śloderbach [1], [2], have been introduced for simplification of interpretation of different terms of effects of couplings occurring in the equations. They can be also useful for some simplifications in general constitutive equations. They are a kind of numbers which take a value 1 or zero when any of effects of couplings shown in Fig. 1 is neglected. For example, when $\gamma_{4}=0$ is substituted, thermal expansion is neglected, if $\gamma_{11}=0-$ a change of internal forces caused by a change of elastic strains is neglected, when $\bar{\gamma}_{6}=0$ - heat of elastic deformations is not taken into account, in the case of $\bar{\gamma}_{9}=0$ - influence of internal changes on elastic strains is omitted, etc. If we are concentrated only on internal changes in the material caused by plastic strains and occuring as plastic hardening, i.e., the effects denoted by numbers 9-12 in Fig.1,they can also be called the effects of elastic-plastic coupling (Hueckel [31], Śloderbach [1], [2], Śloderbach and Pająk 25]).

Theoretically, there are 30 physical kinds of thermodynamic, thermomechanical and mechanical (elastic-plastic) couplings. Not all of them, however, have their physical interpretation and physical explanation, and not all of them have been observed. They are a result of the assumption of a general (nonadditive) form for thermodynamic potentials and formal derivation of total differentials for the chosen dependent parameters of state, depending on the assumed systems of independent parameters of state, see expressions $(3.22)-(3.27)$.

The symbol $\gamma_{0}$ shows dissipation heat which is not a thermostatic thermal effect and it is not specified in the diagram in Fig. 1.

Some of those couplings have already their names and definitions, such as heat capacity, elasticity, thermal expansion, elastic thermal stresses, thermostatic piezoelectric effect, heat of internal changes, heat of elastic strains, internal thermal forces. Other couplings have only formal names resulting from a change of the given physical quantity dependent on the independent parameter, such as a change of internal forces accompanying internal changes, a change of elastic strains caused by internal changes, a change of the stress state caused by internal changes, a change of internal forces caused by a change of the stress state, influence of temperature on internal changes and influence of internal changes on a change of temperature, etc.

\section{Systems of constitutive thermo-elasto-plastic equations}

Eliminating $\dot{s}$ from Eqs (2.3), (3.22) $)_{1,2}$, we obtain two alternative equations for temperature. By finding $\dot{\sigma}$ from Eq.(3.5) $)_{2}$, and $\dot{\varepsilon}^{e}$ from Eq.(3.6) , and making use of Eqs (3.9)-(3.12), respectively, we obtain the following two alternative sets of equations composed of the equation for the temperature and the relation between the rates of elastic strain, elastic stress and temperature

$$
\left\{\begin{array}{l}
\rho_{0} c_{\sigma} \dot{T}=\gamma_{0} D-\gamma_{7} T \alpha: \dot{\sigma}+\bar{\gamma}_{8} T \frac{\partial \Pi}{\partial T} \cdot \dot{K}+q_{0}, \\
\dot{\boldsymbol{\varepsilon}}^{\mathrm{e}}=\gamma_{2} \boldsymbol{L} \dot{\sigma}+\gamma_{4} \alpha \dot{T}+\bar{\gamma}_{9} Z \dot{K} .
\end{array}\right.
$$




$$
\left\{\begin{array}{l}
\rho_{0} c_{\varepsilon} \dot{T}=\gamma_{0} D-\bar{\gamma}_{6} T \boldsymbol{\alpha}: \boldsymbol{M} \dot{\boldsymbol{\varepsilon}}^{\mathrm{e}}+\bar{\gamma}_{8} T \frac{\partial \Pi}{\partial T} \cdot \dot{K}+q_{0}, \\
\dot{\boldsymbol{\sigma}}=\bar{\gamma}_{2} \boldsymbol{M} \dot{\boldsymbol{\varepsilon}}^{\mathrm{e}}-\gamma_{5} \dot{T} \boldsymbol{M} \boldsymbol{\alpha}+\bar{\gamma}_{10} \boldsymbol{N} \dot{K} .
\end{array}\right.
$$

where $q_{0}=-\operatorname{div} \boldsymbol{q}$.

Increment of the temperature is

$$
\Theta=T-T_{0},
$$

where $T_{0}$ - is the temperature of reference $[K]$.

Let us observe that the elastic strain rate involved in Eq.(4.1 $)_{2}$ can be represented in the form

$$
\begin{aligned}
& \dot{\boldsymbol{\varepsilon}}^{e}=\dot{\boldsymbol{\varepsilon}}^{e I}+\dot{\boldsymbol{\varepsilon}}^{e I I}, \\
& \left\{\begin{array}{l}
\dot{\boldsymbol{\varepsilon}}^{e I}=\gamma_{2} \boldsymbol{L} \dot{\boldsymbol{\sigma}}+\gamma_{4} \alpha \dot{T}, \\
\dot{\boldsymbol{\varepsilon}}^{e I I}=\bar{\gamma}_{9} \boldsymbol{Z} \dot{K},
\end{array}\right.
\end{aligned}
$$

where $\dot{\varepsilon}^{e I}$ - is termed the „uncoupled” part of the elastic strain rate, and $\dot{\varepsilon}^{e I I}$ is the "coupled" part of the elastic strain rate connected with the internal processes accompanying the plastic strain. Such a separation of the tensor $\dot{\varepsilon}^{e}$ into two parts was adopted and interpreted in Hueckel [31], Śloderbach [1], [2], [34] in the case of isothermal and nonisothermal processes. By confronting (4.4) with (2.7) it is found that

$$
\dot{\boldsymbol{\varepsilon}}=\dot{\boldsymbol{\varepsilon}}^{e}+\dot{\boldsymbol{\varepsilon}}^{p}=\dot{\boldsymbol{\varepsilon}}^{e I}+\dot{\boldsymbol{\varepsilon}}^{e I I}+\dot{\boldsymbol{\varepsilon}}^{p},
$$

or $\quad d \boldsymbol{\varepsilon}=d \boldsymbol{\varepsilon}^{e}+d \boldsymbol{\varepsilon}^{p}=d \boldsymbol{\varepsilon}^{e I}+d \boldsymbol{\varepsilon}^{e I I}+d \boldsymbol{\varepsilon}^{p}$.

A graphical illustration of such a separation of strains is shown in Figs 2 and 3 for a stress and strain cycle, respectively.

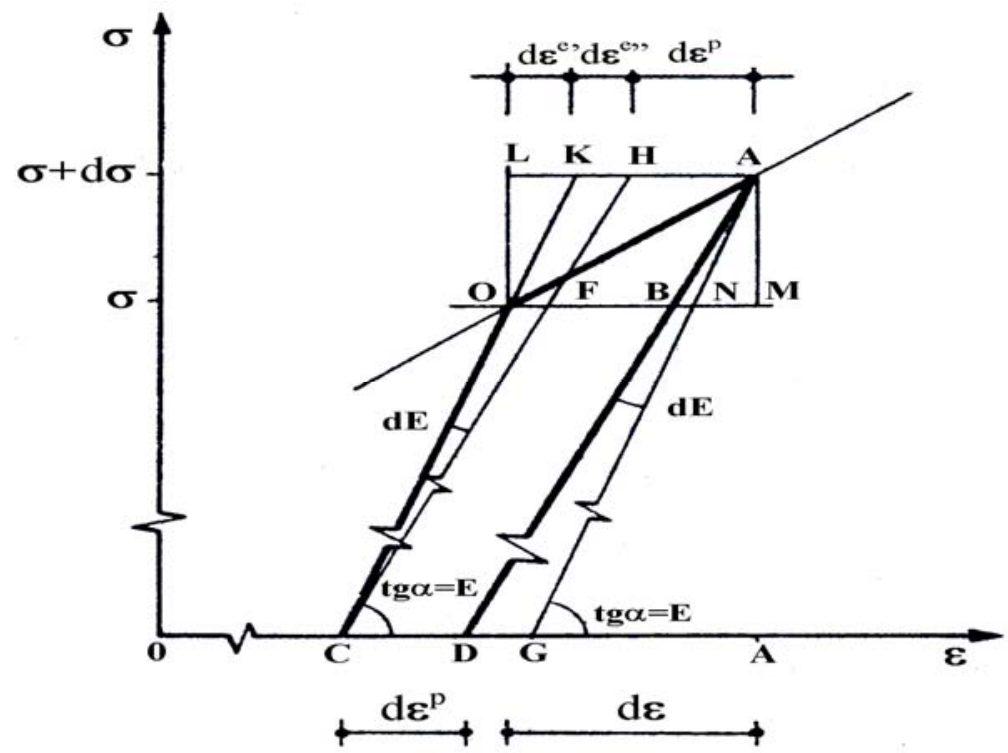

Fig.2. Graphical interpretation of the strain rate tensor distribution. 


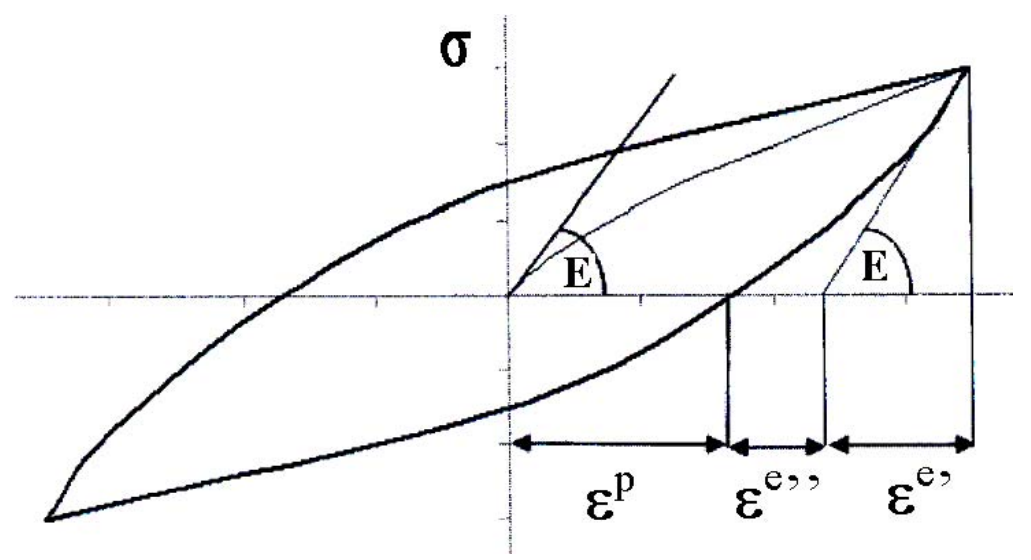

Fig.3. The hysteresis loop and the cyclic strain curve with the selected elastic deformation, "uncoupled" (linear elastic) and "coupled" respectively.

Figures 2 and 3 present linear and nonlinear changes of elastic deformations with the stresses during the unloading processes. Physically, it means a linear and nonlinear change of Young's moduli caused by plastic deformations. In Figs 2 and 3 we can see a decrease of plastic deformations being a result of a decrease of Young's modulus value for the considered materials. An inverse phenomenon (increase of Young's modulus value) seems to be possible for other modern materials.

The closed cycles of stresses and strains play the most important role in problems of mechanical (high-cycle) fatigue (Killer [35]) or thermo-mechanical (low-cycle) fatigue (Coffin [36], Costa and Silva [37], Okrajni et al. [38]). In recent years, a wide research related to thermo-mechanical fatigue (most often to low-cycle fatigue) of many technical devices and their components has been mode (see Baumel and Seegler [39], Coffin [36], Costa and Silva [37], Killer [35], Marcisz et al. [40], Okrajni et al. [38]) for example.

Let us introduce another thermodynamic potential - the enthalpy (Callen [26], Dillon [27], Guminski [28], Kaleta [41], Śloderbach [1], [2], [34], Śloderbach and Rechul [7], Śloderbach and Pająk [8])

$$
\Gamma\left(Y_{K}^{T \sigma}\right)=G\left(Y_{K}^{T \sigma}\right)+T_{S}\left(Y_{K}^{T \sigma}\right) .
$$

The enthalpy potential was efficiently applied in the papers (Kaleta [41], Śloderbach [34], Śloderbach and Rechul [7], Śloderbach and Pająk [25]) for determination of the stored energy of plastic deformations (SEPD) (Bever and Holt [4], Kaleta [41], Oliferuk [6]) in both quasi-static and cyclic loading processes.

Then, making use of Eqs (4.8) and (2.1) $)_{1}$, the equation for the temperature can be expressed in terms of plastic strain power and the variation in enthalpy due to a variation in the internal parameters as follows

$$
\rho_{0} c_{\sigma} \dot{T}=\gamma_{0} \sigma: \dot{\varepsilon}^{p}-\gamma_{7} T \alpha: \dot{\sigma}-\bar{\gamma}_{8} \rho_{0} \frac{\partial \Gamma\left(Y_{K}^{T \sigma}\right)}{\partial K} \cdot \dot{K}+q_{0} .
$$

In this form, Eq.(4.9) can be useful for the analysis of the energy stored in the body as a result of plastic deformation in a closed cycle of stress (Raniecki [12], [13], Śloderbach [34], Śloderbach and Rechul [7], Śloderbach and Pająk [8]). 


\section{Rate equations}

\subsection{General form of the rate equations}

The thermal equations of state appearing in any column of Tab.1, represent the first group of the constitutive equations of thermoplasticity. The other group are the rate equations (Nguyen [24], Raniecki [12], [13], Raniecki and Thermann [16], Śloderbach [1], [2], [34]) relating thermodynamic impulses $\left\{\boldsymbol{\sigma},-\Pi, \frac{1}{T} \nabla T\right\}$ to the thermodynamic flow rate $\left\{\dot{\boldsymbol{\varepsilon}}^{p}, \dot{K}, \boldsymbol{q}\right\}$.

The rate equations will be assumed in the following general form

$$
\mathbf{q}=\varphi^{q}\left(\nabla T, Y_{K}^{T}\right) \quad \text { and } \quad \varphi^{q}\left(0, Y_{K}^{T}\right)=\mathbf{0},
$$

where $T=\operatorname{grad} T$ and $Y_{K}^{T}=\{T, K\}$.

Equations of plastic flow are the following

$$
\dot{\boldsymbol{\varepsilon}}^{p}=\Lambda \frac{\partial F_{1}}{\partial \boldsymbol{\sigma}}, \quad \dot{\mathrm{K}}=\Lambda b(\sigma,-\Pi, T, \mathrm{~K})=\Lambda b \quad \text { or } \quad-\dot{K}=\Lambda \frac{\partial F_{1}}{\partial \Pi},
$$

if

$$
\begin{array}{ll}
F_{l}=0 \quad \text { and } \quad \Lambda \geq 0, & \\
\dot{\varepsilon}^{p}=0, \quad \dot{K}=0 \quad \text { if: } \quad F_{l}<0, \quad \text { or } \quad F_{l}=0 \quad \text { and } \quad \Lambda<0
\end{array}
$$

where $b$ - function describing evolution for internal parameters $\dot{K}$.

Here, $F_{l}=F_{l}(\sigma,-\Pi, T, K)$ - is a generalized function of plastic flow determined in the space of thermodynamic forces $X^{d}=\{\sigma,-\Pi\}$ such that $\left(F_{1}=0\right)$ determines the flow area in this space. The expression for an equation of evolution for the vector of pairs $\dot{K}$ in the form of Eq.(5.2) $)_{2}$ is a result of application of the idea of the preparation space (Perzyna [9]). Using additional experimental data, in that space we can determine the equations of evolution for internal parameters $K$ or dissipation forces $\Pi$ and determine their initial value (Perzyna [9], Śloderbach [34], Śloderbach and Rechul [7], Sloderbach and Pająk $[8])$.

Since $\varphi^{q}$ does not depend on dissipation forces $\{\sigma,-\Pi\}$ two independent inequalities must be fulfilled if the second law of thermodynamics (dissipation rule) must be satisfied (Petryk [30], Raniecki [12], [13], Raniecki and Thermann [16], Śloderbach [1], [2], [34])

$$
-\varphi^{q}\left(\nabla T, Y_{K}^{T}\right) \cdot \nabla T \geq 0 \quad \text { and } \quad \frac{\partial F_{1}}{\partial \sigma}: \dot{\sigma}-\Pi \cdot b \geq 0
$$

If $\Pi$ or $K$ are replaced by a suitable equation of state [columns of Eqs (3.5) or (3.7) from Tab.1], then we will obtain the plastic flow function and suitable flow conditions $F$ in the stress space, written as

$$
F(\sigma, K, T)=\left.F_{I}(\sigma,-\Pi, K, T)\right|_{\Pi=\Pi(\sigma, K, T)},
$$

or

$$
\bar{F}(\sigma,-\Pi, T)=\left.F_{I}(\sigma,-\Pi, K, T)\right|_{K=K(\sigma, \Pi, T)} .
$$


The factor $\Lambda$ in Eq.(5.2) can be eliminated by making use of the ,association condition” (Prager [11], Raniecki [12], [13], Raniecki and Thermann [16], Śloderbach [1], [2], [34], Śloderbach and Rechul [7])

$$
\begin{aligned}
& \dot{F}_{l}=\dot{F}=0 \quad \text { if } \quad F_{l}=F=0 \quad \text { as: } \\
& \frac{\partial F}{\partial \sigma}: \dot{\sigma}+\frac{\partial F}{\partial T} \dot{T}-\Lambda h=0 \quad \text { then: } \quad \Lambda=\left(\frac{\partial F}{\partial \sigma}: \dot{\sigma}+\frac{\partial F}{\partial T} \dot{T}\right) \frac{1}{h}
\end{aligned}
$$

where $\quad h=-\frac{\partial F}{\partial K} \cdot b$,

is what is termed the strain hardening function.

By assuming the classical condition for plastic loading $\left(\dot{\varepsilon}^{p} \neq 0\right)$, if and only if $\frac{\partial F}{\partial \sigma}: \dot{\sigma}+\frac{\partial F}{\partial T} \dot{T} \geq 0$ we find, by virtue of Eq.(5.6) 2 , that $h \geq 0$. Equations (5.2) $)_{1}$, can be expressed in the form

$$
\begin{aligned}
& \dot{\boldsymbol{\varepsilon}}^{p}=\left\{\frac{1}{h} \frac{\partial F_{1}}{\partial \boldsymbol{\sigma}}\left(\frac{\partial F}{\partial \boldsymbol{\sigma}}: \dot{\sigma}+\frac{\partial F}{\partial T} \dot{T}\right) \quad \text { if } \quad F=0 \quad \text { and } \quad \frac{\partial F}{\partial \boldsymbol{\sigma}}: \dot{\sigma}+\frac{\partial F}{\partial T} \dot{T} \geq 0,\right. \\
& \dot{\boldsymbol{\varepsilon}}^{p}=0 \quad \text { if } \quad F<0 \quad \text { or if } \quad F=0 \quad \text { and } \quad \frac{\partial F}{\partial \boldsymbol{\sigma}}: \dot{\sigma}+\frac{\partial F}{\partial T} T<0
\end{aligned}
$$

where, by virtue of Eq.(5.5)

$$
\frac{\partial F}{\partial \sigma}=\frac{\partial F_{1}}{\partial \sigma}+\frac{\partial F_{1}}{\partial \Pi} * \frac{\partial \Pi}{\partial \sigma} .
$$

For the analysis of SEPD during cyclic loadings and for the initial stage of plastic deformations (see Oliferuk [6], Śloderbach [34], Śloderbach and Rechul [7], Śloderbach and Pająk [8]) we can assume the following form of the flow condition (Raniecki [13], Śloderbach [34], Śloderbach and Rechul [7])

$$
F_{l}=\frac{1}{2}(\boldsymbol{S}-c \pi):(\boldsymbol{S}-c \pi)-\frac{1}{3} Y_{1}^{2}(\pi, \kappa, T)=0
$$

where $S=\sigma^{\prime}=\operatorname{dev} \sigma=\sigma-\frac{1}{3}\left(\sigma_{i i}\right) 1, c=c(\kappa)-$ is a material parameter,

$Y_{1}-$ is the yield point under simple tension, and the internal thermodynamic force $\pi$ can be identified with the force resulting in the translatory motion of the flow surface in the space $S$, causing kinematic hardening and the Bauschinger effect (see Pęcherski [33], Raniecki [13], Śloderbach [34], Śloderbach and Rechul [7]), for example.

In the case of quasi-static (non-cyclic) loadings and big strains, isothropic hardening is dominating (Pęcherski [33], Śloderbach [34], Śloderbach and Rechul [7], Śloderbach and Pająk [8]). For such processes of plastic deformations, the following simplified flow condition with isotrophic hardening is assumed (Raniecki [13])

$$
F_{l}=\frac{1}{2} S: S-\frac{1}{3} Y_{1}^{2}(\pi, \kappa, T)=0
$$


In Eq.(5.11) compared with Eq.(5.10) it was assumed that $\pi=0$. Then, it appears that vectors of pairs ( $K$ and $\Pi$ ), are $K=\kappa$ and $\Pi=\pi$ respectively. The flow conditions (5.10) and (5.11) are (H-M-H) conditions. Condition (5.11) describes a special class of materials with isothropic hardening, the internal state of which is described by only one scalar parameter $\kappa$, and irreversible plastic flow is described by the following function of flow

$$
F_{l}=\sigma_{(i)}-Y_{l}(\pi, \kappa, T), \quad \text { where }\left.\quad Y_{l}(\pi, \kappa, T)\right|_{\pi=\pi(\sigma, \kappa, T)}=Y(\sigma, \kappa, T),
$$

or

$$
F_{l}=\sigma_{(i)}-Y_{l}(\pi, \kappa, T), \quad \text { where }\left.\quad Y_{l}(\pi, \kappa, T)\right|_{\kappa=\kappa(\sigma, \pi, T)}=\bar{Y}(\sigma, \pi, T),
$$

and

$$
\left.Y_{l}(\pi, \kappa, T)\right|_{\pi=\pi(\sigma, \kappa, T)}=\left.Y_{l}(\pi, \kappa, T)\right|_{\kappa=\kappa(\sigma, \pi, T)} \quad \text { or } \quad Y(\sigma, \kappa, T)=\bar{Y}(\sigma, \pi, T)
$$

where $\sigma_{(i)}=\left(\frac{3}{2} S: S\right)^{\frac{1}{2}}$ - effective deviator of stress.

In this case, the associated laws of plastic flow Eq.(5.2), for $\left(F_{l}=0\right)$ take the following form (Raniecki [13], Śloderbach [34], Śloderbach and Rechul [7])

$$
\dot{e}^{\mathrm{p}}=\frac{3}{2} \frac{\Lambda}{\sigma_{(\mathrm{i})}} \boldsymbol{S}, \quad \dot{\boldsymbol{\varepsilon}}_{i i}^{p}=\mathbf{0}, \quad \dot{\mathrm{\kappa}}=\Lambda \frac{\partial Y_{1}}{\partial \pi}
$$

where

$$
\dot{\boldsymbol{e}}^{p} \equiv \dot{\boldsymbol{\varepsilon}}^{p}=\operatorname{dev} \dot{\boldsymbol{\varepsilon}}^{p}
$$

The power of plastic deformation has the form

$$
\dot{W}^{p}=\boldsymbol{S}: \dot{e}^{p}=\sigma_{(i)} \dot{e}_{(i)}^{p}
$$

where $\dot{e}_{(i)}^{p}=\left(\frac{2}{3} \dot{\boldsymbol{e}}^{p}: \dot{\boldsymbol{e}}^{p}\right)^{\frac{1}{2}}$ - means the intensity of the plastic strain rate, and $e_{(i)}^{p}=\int_{0}^{t} \dot{e}_{(i)}^{p} d \tau$ - is the intensity of the plastic strain deviator.

\subsection{The particular form of rate equations resulting from the Gyarmati postulate}

A more special form of rate equations can be obtained by making use of the Gyarmati postulate (Gyarmati [32], Raniecki [12], [13], Raniecki and Thermann [16], Śloderbach [1], [2], [34], Śloderbach and Rechul [7]). Let us assume that the dissipative thermodynamic forces $X^{d}$ see Eq.(2.8), have a potential $\Psi^{d}=$ $\Psi^{d}\left(\dot{x}^{d}, Y_{K}^{T}\right)$, (see Gyarmati [32], Raniecki [12], [13], Raniecki and Thermann [16], Śloderbach [34])

$$
X^{d}=\frac{\partial \Psi^{d}}{\partial \dot{x}^{d}}, \quad \dot{x}^{d}=\left\{\dot{\boldsymbol{\varepsilon}}^{p}, \dot{K}\right\}
$$

which is differentiable everywhere except at the point $\dot{x}^{d}=0$. 
Since the relations Eq.(5.15) are to describe a plastic material insensitivity to thermodynamic flow rate, they must be invariant under a change of time scale. It can be easily seen that this is the case, provided that $\Psi^{d}$ is a homogeneous function of the first order in $\dot{x}^{d}$.

The homogeneity of the function $\Psi^{d}$ implies in turn the dependence of the function $X^{d}\left(\dot{x}^{d}, Y_{K}^{T}\right)$ (see e.g., Raniecki [12], [13], Raniecki and Thermann [16], Śloderbach [34]).

This means that there exists a function $F_{l}\left(X^{d}, Y_{K}^{T}\right)$ termed the generalized flow function such that

$$
F_{1}\left(X^{d}, Y_{K}^{T}\right)=0 \quad \text { if } \quad \dot{x}^{d} \neq 0
$$

Then the inverse relations to Eq.(5.15) can be represented in the form

$$
\dot{x}^{d}=\Lambda \frac{\partial F_{1}\left(X^{d}, Y_{K}^{T}\right)}{\partial X^{d}} \quad \text { if } \quad \dot{x}^{d} \neq 0 \quad \text { and } \quad F_{1}=0 .
$$

Assuming that the states of $F_{1}>0$ cannot be attained and taking the usual loading and unloading criterion $(5.8)_{1,2}$ we shall obtain generalized W. Prager's non-isothermal laws of plastic flow (see Raniecki [12], [13], Raniecki and Thermann [16], Śloderbach [1], [2], [34]) in a form which is identical with (5.8) except that the function $b$ involved in Eqs (5.2) 2,3 and (5.7) has now the form

$$
b=-\frac{\partial F_{1}\left(X^{d}, Y_{K}^{T}\right)}{\partial \Pi} .
$$

Making use of Eqs (5.18), (5.9) and expressions (3.11) and (3.19), we find

$$
\Lambda \frac{\partial F}{\partial \boldsymbol{\sigma}}=\dot{\boldsymbol{\varepsilon}}^{p}+\frac{\partial \boldsymbol{\varepsilon}^{e}}{\partial K} * \dot{K}
$$

where $\Lambda$ is defined by the formula $(5.6)_{2}$.

Substituting (4.5) $)_{2}$ into (5.19), we find

$$
\Lambda \frac{\partial F}{\partial \boldsymbol{\sigma}}=\dot{\boldsymbol{\varepsilon}}^{p}+\dot{\boldsymbol{\varepsilon}}^{e I I}
$$

In the general formulation, the tensor $\dot{\boldsymbol{\varepsilon}}^{p}$ is normal to the surface $F_{1}=0$, but is not normal to the surface $F=0$. As regards the sum of tensors $\dot{\boldsymbol{\varepsilon}}^{p}+\dot{\boldsymbol{\varepsilon}}^{e I I}$ it is normal to the surface $F=0$, but is not normal to the surface $F_{1}=0$, where $\dot{\varepsilon}^{e I I}=\frac{\partial F_{1}}{\partial \Pi} * \frac{\partial \Pi}{\partial \sigma}$. As a consequence of the Gyarmati postulate, the sum of tensors $\dot{\boldsymbol{\varepsilon}}^{p}+\dot{\boldsymbol{\varepsilon}}^{e I I}$ is also normal to the surface $F=0$, but now $\dot{\boldsymbol{\varepsilon}}^{e I I}=\frac{\partial \boldsymbol{\varepsilon}^{e}}{\partial K} * \dot{K}$. It seems that this interpretation of this effect has not been observed yet in the literature. It is ilustrated in Fig.4. 


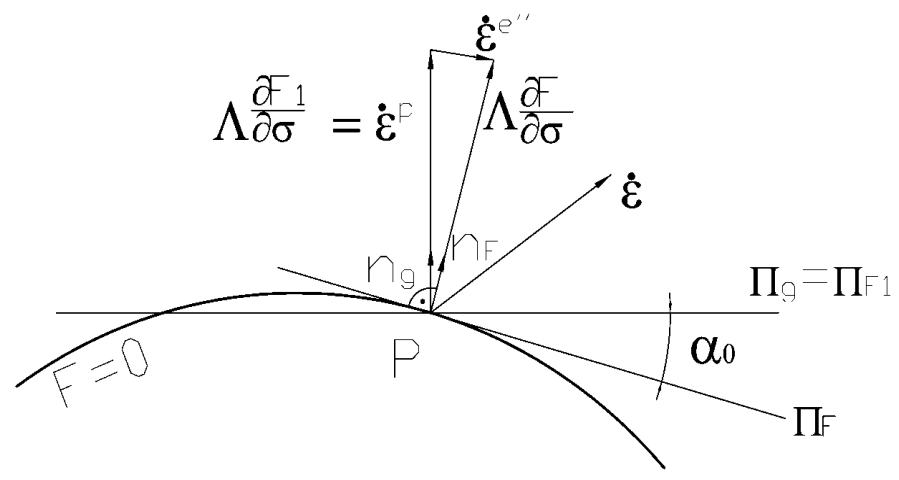

Fig.4. Graphically-vectorial interpretation of expression (3.20).

In Fig.4:

$\prod_{F}$ - denotes the hyperplane tangent to the instantaneous yield surface $F=0$ at the point $P$ determined by the unit normal vector $\boldsymbol{n}_{f}$, where $\boldsymbol{n}_{f}=\frac{\boldsymbol{F}_{\sigma}}{\left(\frac{1}{\left.F_{\sigma} \cdot \boldsymbol{F}_{\sigma}\right)}\right.}$,

$$
\left(\boldsymbol{F}_{\sigma} \cdot \boldsymbol{F}_{\sigma}\right)^{\frac{1}{2}}
$$

$\Pi_{F_{l}}$ - denotes the hyperplane tangent to the instantaneous yield surface $F_{l}=0$ having the character of plastic potential and determined by the normal unit vector $\boldsymbol{n}_{g} \equiv \boldsymbol{n}_{f l}$, where $\boldsymbol{n}_{g}=\frac{\boldsymbol{F}_{l, \sigma}}{1}$ where $\boldsymbol{F}_{\sigma}=\frac{\partial F}{\partial \boldsymbol{\sigma}}$ and $\boldsymbol{F}_{1, \sigma}=\frac{\partial F_{1}}{\partial \boldsymbol{\sigma}}$,

$$
\left(\boldsymbol{F}_{1, \sigma} \cdot \boldsymbol{F}_{1, \sigma}\right)^{\frac{1}{2}}
$$

and $\alpha_{0}-$ is the angular measure of deviation due to the variation in internal foces $\Pi$ depending on the the state of stress (the effect denoted by a symbol $\gamma_{12}$, cf. Sect. 3), which is equivalent to a change in moduli of elasticity as a result of plastic deformation, see Eq.(5.19) $)_{2}$ and coupling effect denoted by a symbol $\gamma_{9}^{*}$, cf. Sect. 3.

In the outline of the theory of material intensitive to thermodynamic flow rate, all the equations take a definite form, even the thermodynamic function $G$ (or any other thermodynamic potential), $\Psi^{d}$ and $\varphi^{q}$ or $G$, $F_{1}$ and $\varphi^{q}$. In the most general formulation given in Sect. 3.1 it is also necessary to know the function $b=b\left(X^{d}, Y_{K}^{T}\right)$ for the equations of evolution of the internal parameters $K$.

\section{Final remarks}

1. In the paper, the authors derive the field equations of coupled generalized thermo-elasto-plasticity using postulates of classical thermodynamics of irreversible processes. The idea of macroscopic internal parameters is used for the description of thermodynamic state of the elastic-plastic body. Derivation of basic relationships is based on some previous papers concerning that area (Dillon [27], Kestin [29], Perzyna and Sawczuk [42], Raniecki [12], [13], Raniecki and Sawczuk [14], [15], Raniecki and Thermann [16], Śloderbach [1], [2], [34], Ziegler [17]).

2. In the paper, the authors use a general form of thermodynamic potentials. An additive form - for example for the free energy potential - is not applied in order to specify a closed system of couplings in thermo-elasto-plasticity. The additive form presents two terms: one of them describes thermoelastic properties, and the other one - thermo-plastic properties. In the case of such separation of the potential into two terms, effects of the elastic-plastic coupling do not occur. The additive form of free 
energy has the form (see e.g., Raniecki [12], [13], Raniecki and Sawczuk [14], [15], Raniecki and Thermann [16])

$$
A=A\left(T, \varepsilon^{\mathrm{e}}, K\right)=A^{e}\left(T, \varepsilon^{\mathrm{e}}\right)+A^{p}(T, K) .
$$

3. The generalization consists in formal derivation, with the use of Legendre transformations, of a new thermodynamic potential dependent on internal thermodynamic forces. Owing to that, full closure of the system of physical thermo-elasto-plastic couplings (thermomechanical and elastic-plastic couplings) was possible. Suitable couplings were presented as a closed diagram of couplings. Derivation of the new thermodynamic potential allowed a precise description of quantities of the stored energy of plastic deformation (SEPD) obtained during experiments, and specification of equations of evolution for internal thermodynamic forces.

4. In the paper it is assumed that gradients of displacements and strain velocities are low, and for simplification it is assumed that all the mathematical operations and all the descriptions are realized in the Cartesian coordinate system.

5. Many papers describing some chosen kinds of thermomechanic couplings including large deformations has been published (Benall and Bigoni [20], Candija and Brnic [21], Casey [22], Epstein and Maugin [43], Lehmann [23], Markin and Sokolova [44], Xiao et al. [45], Nguyen [29], Śloderbach and Pająk [25]). They do not present, however, a full description of a closed set of equations and thermomechanic couplings, and they do not form a closed diagram of couplings.

6. The diagram shown in Fig.1 has got the following recognized properties resulting from a set of thermodynamic identities (3.9)-(3.21) and principles of reciprocity of physical phenomena.

a. Suitable pairs of couplings $(5,6),(8,13)$ and $(9,12)$ (mutually parallel lines) and pairs of couplings $(4,7),(19,11)$ and $(14,15)$ (mutually crossing lines) have the same coupling parameter which sometimes has a different sign $(+)$ or $(-)$ depending on equations in Tab.1.

b. Coupling triangles, for example lines $(2,9,10),(2,11,12)$ have such a property that one coupling is obtained by a composition of two other ones, for example (coupling $9=$ coupling $2,+{ }^{+}$ coupling10) and 11 = coupling $2,,+"$ coupling 12, see identities (3.10)-(3.13) and (3.18)-(3.19). The sign „," concerns composition (,sum”) of couplings. This property concerns also other suitable triangles of couplings.

c. Couplings (1,2 and 3) are heat capacity, elasticity and plasticity, respectively.

d. It seems that the diagram in Fig. 1 can have other properties resulting from mutual couplings of thermo-elasto-plasticity, not recognized so far.

7. Equations of evolution were derived for internal parameters according to a general approach (with no use of potentials for flows - thermodynamic streams) and an idea resulting from the assumption of the Gyarmati postulate.

8. During derivation of general rate equations of thermo-plasticity, the widely used postulate on the existence of thermodynamic potential (Gyarmati postulate) was not applied. Thus, like in the papers (Hueckel [31], Śloderbach [1], [2], [34]), constitutive equations of plastic flow are non-associated laws even in a special case when the Gyarmati postulate is assumed.

9. The application of the Gyarmati postulate does not have to lead to associated laws of plastic flow. It appears that its application causes that the direction of the sum of tensors of the plastic strain rate and the rate of irreversible strains caused by plastic deformations (the effect of elastic-plastic coupling) is perpendicular to the instantaneous area of plasticity. The existence of the elastic-plastic coupling effect allows the use of the derived equations for describing the behaviour of not only metallic materials, but porous materials, sinters, rocks and soils as well.

10. In the paper, it is assumed that the gradients of displacements and strain rates are low, and for simplicity it is assumed that all the mathematical operations and all the descriptions are realized in the Cartesian coordinate system. In last years, many papers describing some chosen kinds of thermomechanic couplings including large deformations have been published (Candija and Brnic [21], Casey [22], Lehmann [23], Nguyen [24], Pęcherski [33], Śloderbach and Pajak [25], Markin and Sokolova [44], Xiao et al. [45]). 


\section{Nomenclature}

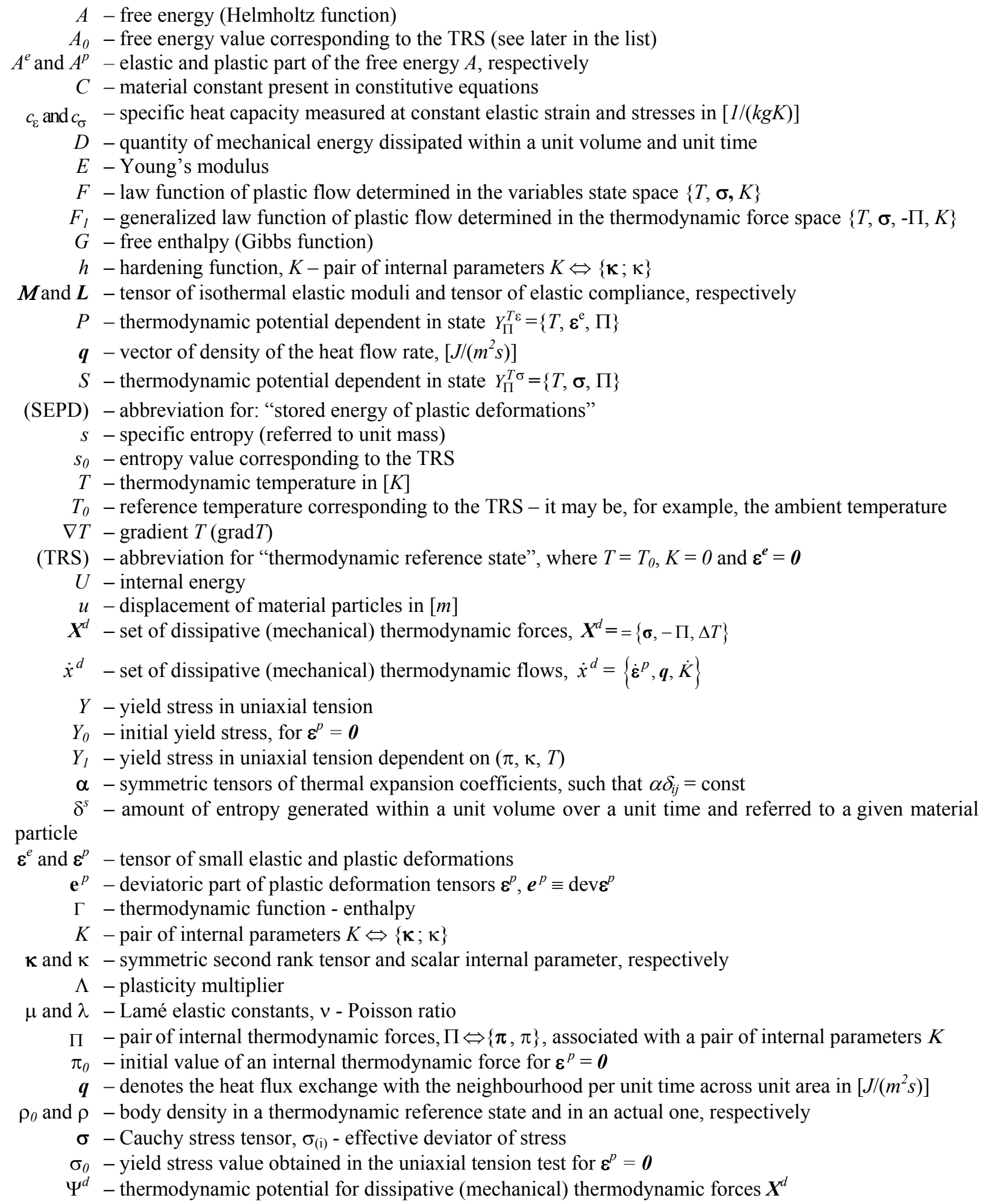




\section{Symbols and abbreviations}

Tensors will be printed in a bold type-face. The summation convention is assumed along with the following detailed notation

$$
\begin{aligned}
& \boldsymbol{A} \boldsymbol{B} \Leftrightarrow A_{i j} B_{j} \quad \text { or } \quad A_{i j k l} B_{k l}(i, j, k, l, m, n, \ldots=1,2,3), \\
& \operatorname{tr} \mathbf{A} \Leftrightarrow A_{k k}, \operatorname{tr}(\boldsymbol{A B}) \Leftrightarrow A_{i j} B_{j i}, \\
& \boldsymbol{A}: \boldsymbol{B} \Leftrightarrow A_{i} B_{i} \quad \text { or } \quad A_{i j} B_{i j} \quad \text { and } \quad \frac{\partial \boldsymbol{A}}{\partial \boldsymbol{B}} d \boldsymbol{B} \Rightarrow \frac{\partial A_{i j}}{\partial B_{k l}} d B_{k l},
\end{aligned}
$$

1 - identity tensor, $\delta_{i j}$ - Kronecker delta, $\boldsymbol{O}$ - null tensor, $\operatorname{sym} \boldsymbol{A} \Rightarrow \frac{1}{2}\left(A_{i j}+A_{j i}\right), \operatorname{dev} \boldsymbol{A}=\boldsymbol{A}-\frac{1}{3}(\operatorname{tr} \boldsymbol{A}) \boldsymbol{1}-$ deviatoric part of tensor $\boldsymbol{A}$,

$$
A_{i, j}=\frac{\partial A_{i}}{\partial x_{j}} \text {, where: } x_{j} \text { - coordinates of a material particle, } \dot{A}=\frac{\partial A}{\partial t} \text {, where: }(t-\text { time }) \text {. }
$$

If $\boldsymbol{Z}$ denotes pairs of tensors of the fourth and the second order, then $\boldsymbol{Z} \Leftrightarrow\left\{Z_{m n k l} ; Z_{m n}\right\}$, and $\boldsymbol{M}$ is the tensor of the fourth order, then the operation $\boldsymbol{M Z}$ is a pair of tensors of the fourth and the second order defined as follows

$$
\boldsymbol{M Z} \Leftrightarrow\left\{M_{i j m n} Z_{m n k l} ; M_{i j m n} Z_{m n}\right\}
$$

If $\Pi$ and $K$ denote pairs of tensors of the second and the zeroth order, then the operation $\Pi \cdot K$ produces a scalar, sec (Śloderbach [1], [2], [3], Śloderbach and Pająk [25])

$$
\Pi \cdot K=\pi: \kappa+\pi \kappa=\pi_{i j} \kappa_{i j}+\pi \kappa .
$$

If the function $F$ is relative to $\Pi$ and $K$, then $F(\Pi)=F(\pi, \pi)$ and $F(K)=F(\kappa, \kappa)$.

The derivatives of function $F$ with respect to a pair $\Pi$ and $K$ are defined as follows

$$
\frac{\partial F}{\partial \Pi}=\left\{\frac{\partial F}{\partial \pi}, \frac{\partial F}{\partial \pi}\right\} \quad \text { and } \quad \frac{\partial F}{\partial K}=\left\{\frac{\partial F}{\partial \kappa}, \frac{\partial F}{\partial \kappa}\right\} .
$$

The differentials of function $F$ with respect to the pairs $K$ and $\Pi$ of tensors of the second and the zeroth order produce the following form

$$
\frac{\partial F}{\partial \Pi} \cdot d \Pi=\left\{\frac{\partial F}{\partial \pi_{i j}} d \pi_{i j}, \frac{\partial F}{\partial \pi} d \pi\right\} \quad \text { and } \quad \frac{\partial F}{\partial K} \cdot d K=\left\{\frac{\partial F}{\partial \kappa_{i j}} d \kappa_{i j}, \frac{\partial F}{\partial \kappa} d \kappa\right\} .
$$

The differential of function $F$ with respect to the pair $K$ of tensors of the second and the zeroth order, produces a sum, (cf. Śloderbach [1], [2], [3], [34], Śloderbach and Pajak [25]), such that

$$
\frac{\partial \Pi}{\partial K} d K \Leftrightarrow\left\{\frac{\partial \pi_{i j}}{\partial \kappa_{k l}} d \kappa_{k l}+\frac{\partial \pi_{i j}}{\partial \kappa} d \kappa ; \frac{\partial \pi}{\partial \kappa_{k l}} d \kappa_{k l}+\frac{\partial \pi}{\partial \kappa} d \kappa\right\} .
$$


If $\alpha$ is a second order tensor, then the operation $\alpha \circ(\boldsymbol{M Z})$ produces a pair of tensors of the second and the zeroth order

$$
\alpha \circ(\boldsymbol{M Z}) \Leftrightarrow\left\{\alpha_{i j} M_{i j m n} Z_{m n k l} ; \alpha_{i j} M_{i j m n} Z_{m n}\right\}
$$

If $\boldsymbol{Z}$ denotes pairs of tensors of the fourth and the second order and $K$ is a pair of tensors of the second and the zeroth order, then the operation $Z * K$ produces a pair of tensors of the second order $s$

$$
\boldsymbol{Z} * K \Leftrightarrow\left\{Z_{i j m n} \kappa_{m n} ; Z_{i j} \kappa\right\}
$$

\section{References}

[1] Śloderbach Z. (1980): Bifurcations criteria for equilibrium states in generalized thermoplasticity. - Doctor's Thesis [in Polish], IFTR-Report, No 37/1980, Institute of Fundamental Technological Research, Polish Academy of Sciences, Warsaw, pp.1-100.

[2] Śloderbach Z. (1983): Generalized Coupled Thermoplasticity. Part I. Fundamental Equations and Identities. Archives of Mechanics, vol.35, pp.337-349.

[3] Śloderbach Z. (1983): Generalized Coupled Thermoplasticity. Part II. On the Uniqueness and Bifurcations criteria. - Archives of Mechanics, No.35, vol.3, pp.351-367.

[4] Bever M., Holt D.L. and Titchener A.L. (1973): Stored Energy of Cold Work. - Progress in Material Sciences, vol.17, Pergamon Press.

[5] Oliferuk W., Korbel A. and Grabski M.W. (1996): Mode of deformation and the rate of energy storage during uniaxial tensile deformation of austenitic steel. - Materials Science and Engineering, A 220, Elsevier Science S.A., pp. $123-128$

[6] Oliferuk W. (1997): Energy storage process and its relation to material structure in austenit steel tested in simple tension [in Polish]. - Habilitation Thesis, IFTR-Reports, No 11/1997, Institute of Fundamental Technological Research, Polish Academy of Sciences, Warsaw, pp.1-108.

[7] Śloderbach Z. and Rechul Z. (2006): A thermodynamic approach to the stored energy concept. - Journal of Technical Physics, vol. XLVII, 2, pp.83-102.

[8] Śloderbach Z. and Pająk J. (2013): Stored energy of plastic deformations in tube bending processes. - International Journal of Applied Mechanics and Engineering, vol.18, No.1, Zielona Gora, pp.235-248.

[9] Perzyna P. (1978): Thermodynamics of inelastic materials [in Polish]. - Warsaw: PWN.

[10] Lehmann Th. (1972): Some thermodynamic considerations of phenomenological theory of non-isothermal elasticplastic deformations. - Archives of Applied Mechanics, vol.24, pp.975-989.

[11] Prager W. (1958): Non-isothermal plastic deformation. - Proceedings Konikladen Nederladen Akademie Wetenschap., B61, pp.176-182.

[12] Raniecki B. (1976): An Introduction to the Concept of Applied Thermoplasticity. - Material Center, Royal Institute of Technology, Stockholm, pp.1-96.

[13] Raniecki B. (1977): Problems of Applied Thermoplasticity. - Habilitation Thesis [in Polish], in IFTR-Reports, No.29/1977, Institute of Fundamental Technological Research, Polish Academy of Sciences, Warsaw, pp.1-120.

[14] Raniecki B. and Sawczuk A. (1973): Thermal effects in plasticity. Part I: Coupled theory. - Zeitschrift für Angewandte Mathematik und Mechanik-ZAMM, vol.55, pp.232-241.

[15] Raniecki B.and Sawczuk A. (1973): Thermal effects in plasticity. Part II: Uniqueness and applications. Zeitschrift für Angewandte Mathematik und Mechanik-ZAMM, vol.55, pp.363-373. 
[16] Raniecki B. and Thermann K. (1978): Infinitesimal Thermoplasticity and Kinematics of Finite Elastic-Plastic Deformations. Basic Concepts. - Mitteilungen aus dem Institut fur Mechanik, No.2, Ruhr-Universitat, Bochum.

[17] Ziegler H. (1963): Some Extremum Principles in Irreversible Thermodynamics with Application to Continuum Mechanics. - Progress in Solid Mechanics, North Holland, Amsterdam, pp.91-193.

[18] Nye J.F. (1957): Physical Properties of Crystals. - Oxford: Clarendon Press.

[19] Newnham R.E. (2005): Properties of Materials. - New York: Oxford University Press Inc..

[20] Benall A. and Bigoni D. (2004): Effects of temperature and thermo-mechanical couplings on material instabilities and strain localization of inelastic materials. - Journal of Mechanics and Physics of Solids, vol.52, No.3, pp.725753.

[21] Candija M. and Brnic J. (2004): Associative coupled thermoplasticity at finite strain with temperature-dependent material parameters. - International J. of Plasticity, vol.20, pp.1851-1874.

[22] Casey J. (1998): On elastic-thermo-plastic materials at finite deformations. - International Journal of Plasticity, vol.14, pp.173-191.

[23] Lehman Th. (1991): Thermodynamical foundations of large inelastic deformations of solids bodies including damage. - Inernational Journal of Plasticity, vol.7, pp.79-98.

[24] Nguyen Huu Viem (1999): Thermomechanical Couplings in Elasto-Plastic Metals in the Case of Finite Deformations [in Polish]. - Habilitation Thesis, IFTR-Reports, No.10/1999, Institute of Fundamental Technological Research, Polish Academy of Sciences, Warsaw, pp.1-116.

[25] Śloderbach Z. and Pająk J. (2010): Generalized coupled thermoplasticity taking into account large strais: Part I. Conditions of uniqueness of the solution of boundary-value problem and bifurcation criteria. - Mathematics and Mechanics of Solids, vol.15, No.3, pp.308-327.

[26] Callen H.B. (1960): Thermodynamics. - N.Y.-London: Edition John Wiley and Sons.

[27] Dillon O.W. (1967): A thermodynamic basis of plasticity. - Acta Mechanica, vol.3, pp.182-195.

[28] Gumiński K. (1974): Thermodynamics. - Warsaw: PWN.

[29] Kestin J. (1973): A Meaning of Thermodynamic in Thermoplasticity. - Vol. „Thermoplasticity”, Ossolineum, Wrocław, pp.5-71.

[30] Petryk H. (1995): Thermodynamic stability of equilibrium in plasticity. - Journal of Non-Equilibrium Thermodynamics, vol.20, pp.132-149.

[31] Hueckel T. (1976): Coupling of elastic and plastic deformations of bulk solids. - Meccanica, vol.11, pp.227-235.

[32] Gyarmati J. (1970): Non-Equilibrium Thermodynamics, Field Theory and Variational Principles. - Berlin-N.Y: Springer.

[33] Pęcherski R. (1998): Description of Plastic Deformations of Metals with Microband of Shearing. - Habilitation Thesis [in Polish], IFTR-Report, No.2/1998, Institute of Fundamental Technological Research, Polish Academy of Sciences, Warsaw, pp.1-132.

[34] Śloderbach Z. (2002): Some Problems of Mechanics in Pipeline Bending Processes [in Polish]. - Publishing House, Wrocław University of Technology, Wroclaw, pp.1-218.

[35] Killer K.J. (1991): An Introductory Lecture on Fatique of Metal. Course of Metal Fatique. - University of Sheffield, England.

[36] Coffin L.F. (1974): Fatique at High Temperature Prediction and Interpretation. - Institution of Mechanical Engineers, vol.188.

[37] Costa N. and Silva F.S. (2011): On a new temperature factor to predict the fatigue limit at different temperatures. - International Journal of Fatigue, vol.33, pp.624-631.

[38] Okrajni J., Junak G. and Marek A. (2008): Modelling of deformation process under thermo-mechanical fatique conditions. - International Journal of Fatique, vol.30, pp.324-329. 
[39] Baumel A. and Seegler T. (1990): Material Data for Cyclic Loading. - Materials Science Monographs, vol.42A-E, Supplement 1, Elsevier Science Publishers.

[40] Marcisz E., Niesłony A. and Łagoda T. (2012): Concept of fatique for determining characteristics of materials with strengthening. - Materials Science Forum, vol.726, pp.43-48.

[41] Kaleta J. (1998): Experimental Foundations for Formulation of Fatique Hypotesis [in Polish]. - Institute of Materials Science and Technical Mechanics, Wrocław University of Technology, No.54, Monographie Series No.24, Wrocław, pp.1-144.

[42] Perzyna P. and Sawczuk A. (1973): Problems of Thermoplasticity. - Nuclear Engineering Design, vol.24, pp.1-55.

[43] Epstein M. and Maugin G.A. (2000): Thermomechanics of volumetric growth in uniform bodies. - International Journal of Plasticity, vol.16, No.7-8, pp.951-978.

[44] Markin A.A. and Sokolova M.Y. (2002): Thermomechanical models of irreversible finite deformation of anisotropic solids. - Strength of Materials, vol.34, No.6, pp.529-535.

[45] Xiao H., Bruhns O.T. and Meyers A. (2007): Thermodynamic laws and consistent Eulerian formulation of finite elastoplasticity with thermal effects. - Journal. of Mechanics and Physics of Solids, vol.55, No.2, pp.338-365.

Received: January 12, 2016

Revised: March 16, 2016 